\title{
Optimization of Energy Consumption in Wireless Sensor Networks using Particle Swarm Optimization
}

\author{
Madhusmita Nandi \\ School of Electronics Engineering, KIIT University \\ Bhubaneswar-751024, Odisha, India
}

\author{
Jibendu Sekhar Roy \\ School of Electronics Engineering, KIIT University \\ Bhubaneswar-751024, Odisha, India
}

\begin{abstract}
The present work is to optimize energy consumption in wireless sensor networks for multi-layer medium access control (ML-MAC) scheme where the delay times, i.e., maximum response time delay, packet transmission delay, clock-drift delay, sleep delay and queuing delay are considered. Particle swarm optimization (PSO) is a popular multidimensional optimization procedure which is used in this paper to achieve less power consumption in wireless sensor networks.
\end{abstract}

\section{Keywords}

Energy consumption, Medium access control, Wireless sensor networks, Particle swarm optimization.

\section{INTRODUCTION}

A wireless sensor network (WSN) is a collection of large number of sensor nodes that are deployed in an ad-hoc manner and communicate using a short-range radio channel. Sensor nodes are battery operated and normally they cannot be recharged due to its deployment in harsh and remote environment. Therefore, energy efficiency is a very critical issue to enhance the network lifetime $[1,2,3]$. ML-MAC is a distributed contention-based MAC algorithm where nodes discover their neighbors based on their radio signal level [48]. Also, ML-MAC is a self-organizing MAC algorithm that does not require a central node to control the operation of the nodes. ML-MAC saves energy to re-send the falsified packets. ML-MAC is a contention based algorithms, which use an active/sleep routine in frame to save energy consumption. There are four major sources of energy waste [1]: collision, overhearing, control packet overhead and idle listening. The frame length $\mathrm{T}_{\text {frame }}$ comprises of the listen and sleep routine. It describe the duty cycle as $\mathrm{T}_{\text {listen }} / \mathrm{T}_{\text {frame }}, \mathrm{T}_{\text {listen }}$ is the active/listen time of a cycle.

In this paper PSO is applied to optimize energy consumption in wireless sensor networks under ML-MAC scheme. Here in existing MAC protocol, listening period is divided into different layers and different types of delays are considered. The optimized results are compared with the results obtained without PSO.

\section{PARTICLE SWARM OPTIMIZATION}

PSO is a robust stochastic optimization procedure based on the movement and intelligence of swarms which is developed by James Kennedy (social-psychologist) and Russell Eberhart (electrical engineer) in $1995[9,10]$. It consists of a swarm of $\mathrm{S}$ candidate solutions called particles. A particle 'i' occupies position $X_{\text {id }}$ and velocity $V_{\text {id }}$ in the dth dimension of the hyperspace, $1 \leq \mathrm{i} \leq \mathrm{S}$ and $1 \leq \mathrm{d} \leq \mathrm{n}$. Each particle is evaluated through an objective function $f(x 1, x 2, x 3, \ldots \ldots \ldots \ldots x n)$, where $f: R^{n} \rightarrow R$. Each particle tries to modify the current positions, the current velocities, the distance between the current position, $\mathrm{P}_{\text {best }}$, the distance between the current position and the $\mathrm{G}_{\text {best }}$. Figure 1 shows the basic concept of PSO lies in accelerating each particle toward its $P_{\text {best }}$ and the $G_{\text {best }}$ locations, with a random weighted acceleration at each time step.

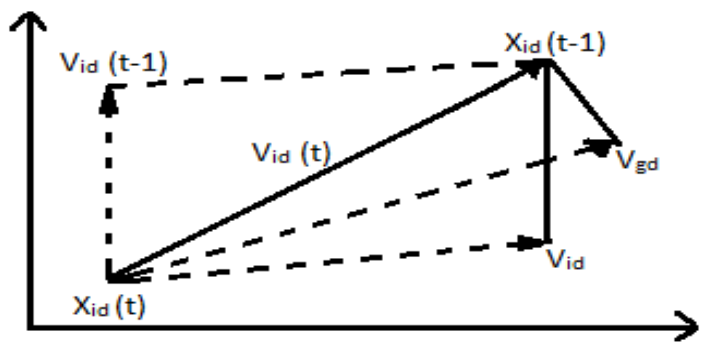

Figure 1: Concept of modification of a searching point by PSO

Steps involved in PSO are given below:

Step 1: Initialize the position and velocity of each particle.

Step 2: Calculate the fitness value of each particle.

Step 3: For each particle, compare the fitness value with the fitness value of $\mathrm{P}_{\text {best }}$, If current value is better, then renew the position with current position, and update the fitness value.

Step 4: Evaluate the best particle of group with the best fitness value. If it is better than fitness value of the $G_{\text {best }}$, then update the $\mathrm{G}_{\text {best }}$ and its fitness value with the position.

Step 5: Check the finalizing criteria, If it is satisfied, quit the iteration; otherwise, return to step 2.

In order to follow the procedure of PSO, one should calculate the value of $X_{i d}(t)$ and $V_{i d}(t)$ by the following equation.

$$
\begin{aligned}
& \mathrm{V}_{\mathrm{id}}(\mathrm{t})=\omega \times \mathrm{V}_{\mathrm{id}}(\mathrm{t}-1) \times \mathrm{C}_{1} \times \phi_{1}\left(\mathrm{P}_{\mathrm{id}}-\mathrm{X}_{\mathrm{id}}(\mathrm{t}-1)\right)+ \\
& \mathrm{C}_{2} \times \phi_{2}\left(\mathrm{P}_{\mathrm{gd}}-\mathrm{X}_{\mathrm{id}}(\mathrm{t}-1)\right) \\
& \mathrm{X}_{\mathrm{id}}(\mathrm{t})=\mathrm{X}_{\mathrm{id}}(\mathrm{t}-1)+\mathrm{V}_{\text {id }}(\mathrm{t})
\end{aligned}
$$

List of parameters are used in equation (1) and (2) are, $\mathrm{V}_{\text {id }}(\mathrm{t})$ : Velocity of particle $i$ at iteration $t, X_{i d}(t)$ : Current position of particle $\mathrm{i}$ at iteration $\mathrm{t}, \mathrm{P}_{\mathrm{id}}$ : Particle's best position, $\mathrm{P}_{\mathrm{gd}}$ : Global best position, $\mathrm{C}_{1}, \mathrm{C}_{2}$ : Learning factors, $\phi_{1}, \phi_{2}$ : Random numbers uniformly distributed in 0 and $1, \omega$ : Inertia weight. The flowchart for PSO is shown in Figure 2. 


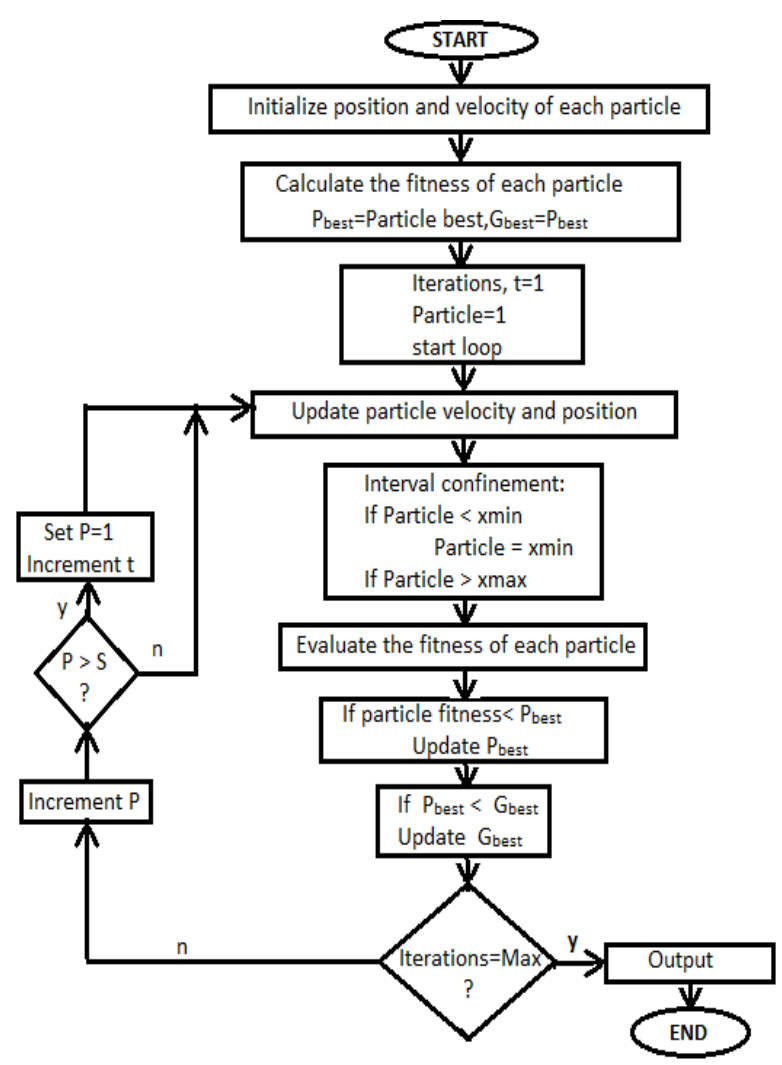

Figure 2: Flow chart of Particle Swarm Optimization

\section{DESIGN OF ENERGY-AWARE ML- MAC ALGORITHM FOR WSN}

A multi-layer MAC (ML-MAC) algorithm is a procedure to reduce node power consumption. The time in ML-MAC is split into frames and each frame is split into two periods: listen and sleep. The active period is sub-split into $\mathrm{L}$ non overlapping layers. Nodes are distributed among the set of layers where nodes in each layers follow a listen/sleep schedule that is skewed in time compared to the schedules of the other layers. A node in ML-MAC algorithm wakes up only at its allocated layer. Therefore, ML-MAC requires less amount of energy because the listen period of a node is short. So there are three main advantages of adopting multiples layers in ML-MAC, i.e., Reduced energy consumption, Low average traffic, Extended network lifetime.

Steps for ML-MAC algorithm are:

Step-1 : The nodes are distributed into different layers using Uniform distributed function.

Step-2 : Then traffic for each node in layers is generated according to a shifted Poisson's distribution function.

Step-3 : Schedule is defined and it is dynamically changed according to the traffic in each layer of the frame conditions.

Step-4: If the sender and receiver nodes are in the same layer then no change has been made to scheduling otherwise the sender has to locate in the layer of the receiver. Hence has to wake in two layers in same active period.

Step-5 : In this model first find which layer of the frame has the least amount of traffic. Then it changed the schedule of the receiver and sender node such that they will both wake in the layer of the frame with least traffic.
Step-6 : Traffic is calculated using distribute matrix (nodes, layers, frames).Then nodes are ready to transmit packets in the layers.

Step-7 : Sender does not have to wake twice in the same period. There will be less collision.

ML-MAC process flow is shown in Figure 3.

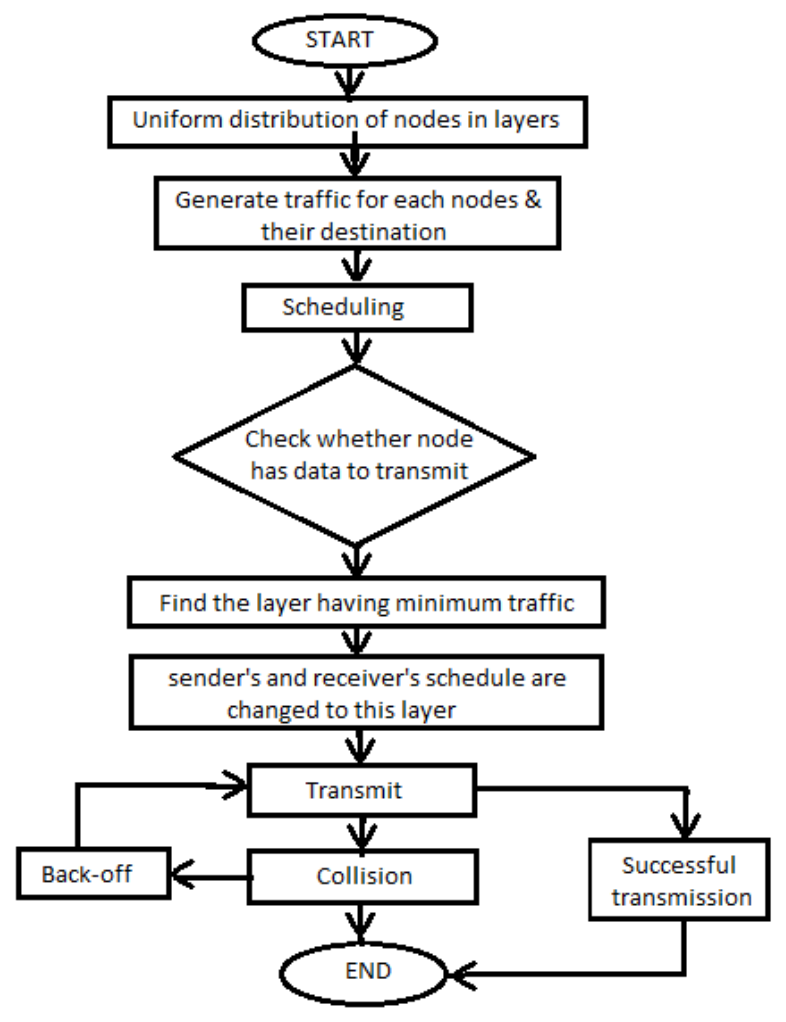

Figure 3: ML-MAC process flow

The design parameters [11] that need to be analyzed to study the achievement of ML-MAC. It has the following design specifications as shown in Figure 4 and Figure 5. Where, n: Total number of nodes in the network, $\mathrm{T}_{\mathrm{N}}$ : Network lifetime, $\mathrm{N}_{\mathrm{F}}$ : Number of fames, L: Number of access layers, $\mathrm{t}_{2}$ : Guard time between layers, $\mathrm{T}_{\mathrm{F}}$ : Frame duration, $\mathrm{t}_{1}$ : Layer duration, $\mathrm{T}_{\mathrm{R}}$ : Maximum response time, $\tau_{\mathrm{\rho}}$ : Propagation delay, $\tau_{\mathrm{d}}$ : Clock drift delay, $\tau_{\mathrm{t}}$ : Packet transmission delay.

There are some assumptions for the behavior of ML-MAC is given below.

- A sensor node generates packets that should follow Poisson distribution function.

- Time is divided into frames and each frame is composed of listen and sleep periods.

- There are three routines for each node, i.e., transmit, sleep and listen.

- Nodes have limited transmit and receive buffer sizes. So the packets will not be dropped as they are all ultimately going to be sent to their destinations.

- $\quad$ All MAC operations are based on the IEEE 802.11.

- There should no bandwidth constraint in the wireless channel.

- TR 1000 is the radio transceiver of the from RF monolithic [12].

Design parameters are given in Table 1. 
Table 1. Parameters are assumed for simulation

\begin{tabular}{|c|c|}
\hline Parameter & Value \\
\hline Average packet inter-arrival time, $\mathrm{T}$ & $2-10 \mathrm{~s}$ \\
\hline Number of layers, $\mathrm{L}$ & $1-10$ \\
\hline Number of nodes, $\mathrm{n}$ & 100 \\
\hline Frame duration, $\mathrm{T}_{\mathrm{F}}$ & $1 \mathrm{~s}$ \\
\hline Layer duration, $\mathrm{t}_{1}$ & $0.3 / \mathrm{L} \mathrm{s}$ \\
\hline Node sleeping power & $15 \mu \mathrm{W}$ \\
\hline Node listening power & $13.5 \mathrm{~mW}$ \\
\hline Node transmitting power & $24.75 \mathrm{~mW}$ \\
\hline Number of initial reservation slots, $\mathrm{W}$ & 8 \\
\hline Node transmission data rate & $19.2 \mathrm{kbps}$ \\
\hline Average packet length, $\alpha$ & $38 \mathrm{Bytes}$ \\
\hline Simulation time & $200 \mathrm{~s}$ \\
\hline
\end{tabular}

The ML-MAC design procedure may be described as [11].

\section{Step-1 : Calculating the frame duration $T_{F}$}

Maximum response time delay $T_{R}$ that is governed by the time to respond the events, the frames duration $\mathrm{T}_{\mathrm{F}}$ is bounded by:

$\mathrm{T}_{\mathrm{F}}>\mathrm{T}_{\mathrm{R}}$

For all layers, $\mathrm{T}_{\mathrm{F}}$ is bounded by total listening time:

$\mathrm{T}_{\mathrm{F}}>\mathrm{t}_{1} \times \mathrm{L}$

Where $t_{1}$ is the listening period per layer which is evaluated in step 2:

Thus from equation (3) and (4), it is bounded as:

$\frac{T_{N}}{T_{R}} \leq N_{F}<\frac{T_{N}}{t_{1}}$

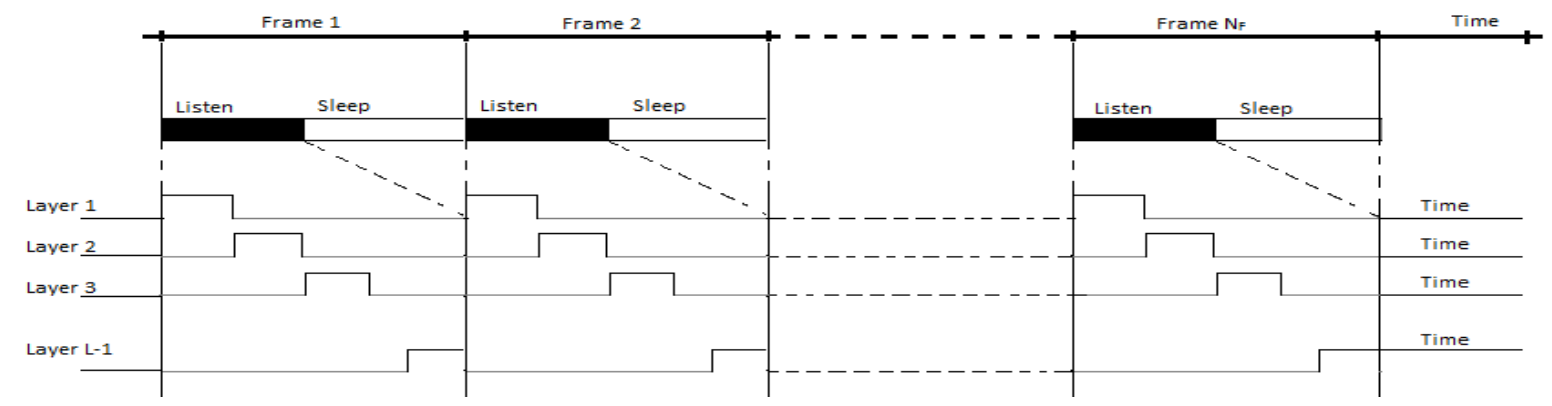

Figure 4: Design overview of ML-MAC

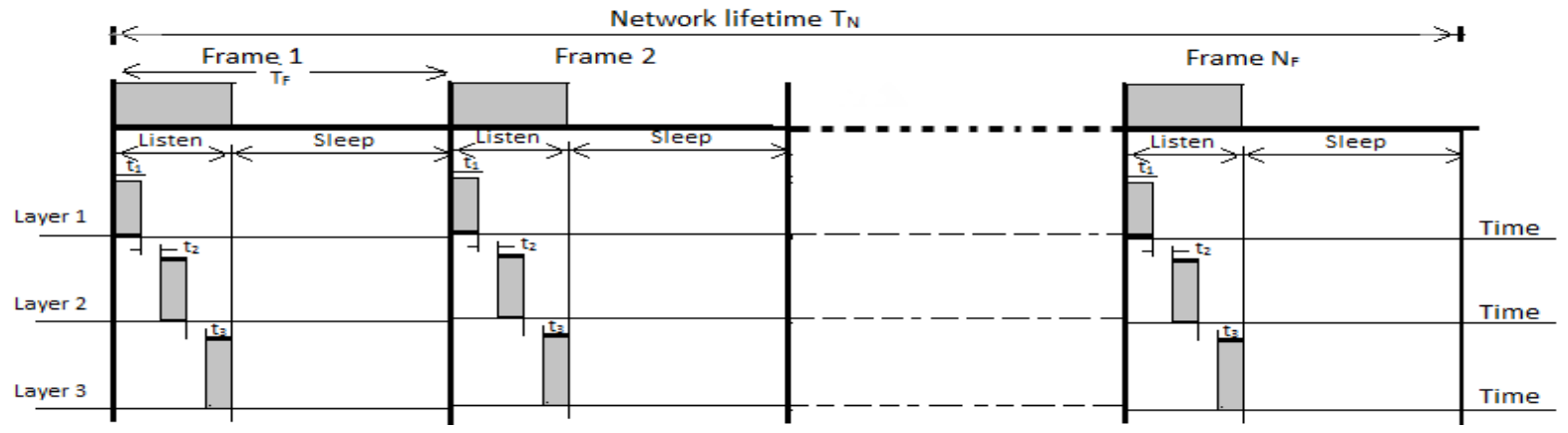

Figure 5: Timing parameters of ML-MAC and Network lifetime $T_{N}$ is split into $N_{F}$ frames
Step-2 : calculating the listening period per layer $t_{1}$

The listening period of one layer $t_{1}$ is governed by the battery capacity $\mathrm{C}$ (mAh : mili ampere hour) and the average node power consumption $\rho$ :

$\rho \times t_{1} \times N_{F} \leq \mathrm{C} \times \mathrm{V}$

Where $\mathrm{V}$ is the average output voltage of the battery. From equation (6), $t_{1}$ is bounded as:

$t_{1} \leq \frac{C \times V}{\rho \times N_{F}}$

Also $t_{1}$ is bounded by the time needed to send at least one packet which is given by following equation:

$\mathrm{t}>\tau_{\mathrm{t}}+\tau_{\rho}+2 \tau_{\mathrm{d}}+\mathrm{W} \tau_{\rho}$

$\mathrm{t}_{1}+\tau_{\rho}+2 \tau_{\mathrm{d}}+\mathrm{W} \tau_{\rho}<\mathrm{t}_{1} \leq \frac{\mathrm{C} \times \mathrm{V}}{\rho \times \mathrm{N}_{\mathrm{F}}}$

Step-3 : estimating the number of layers $L$

The number of layers determine by the average traffic generated per frame which is given by the below equation :

$\lambda_{\text {avg }}=\mathrm{n} \times \lambda \times \mathrm{T}_{\mathrm{F}}$

So, the total listen time should be greater than the time needed to send the entire packet generated by the nodes:

$\mathrm{L} \times \mathrm{t}_{1}>\lambda_{\text {avg }} \times\left(\tau_{\mathrm{t}}+\tau_{\rho}+2 \tau_{\mathrm{d}}+\frac{\mathrm{w}}{2} \tau_{\rho}\right)$

From equation (11) $\mathrm{L}$ is bounded as given below:

$\mathrm{L} \geq \frac{\lambda_{\mathrm{avg}} \times\left(\tau_{\mathrm{t}}+\tau_{\rho}+2 \tau_{\mathrm{d}}+(\mathrm{W} / 2) \tau_{\rho}\right)}{\mathrm{t}_{1}}$

And the guard time between layers $t_{2}$ is governed by the inequality :

$t_{2}>\tau_{\rho}+2 \tau_{d}$

Therefore, the upper limit in $\mathrm{L}$ is given as:

$\mathrm{L}\left(\mathrm{t}_{1}+\mathrm{t}_{2}\right) \leq \mathrm{T}_{\mathrm{F}}$

To get the best behavior, it should determine the values of all timing parameters and the number of layers by using the delay limitations and buffer size in the node. $\frac{\lambda_{\text {avg }} \times\left(\tau_{\mathrm{t}}+\tau_{\rho}+2 \tau_{\mathrm{d}}+(\mathrm{W} / 2) \tau_{\rho}\right)}{\mathrm{t}_{1}} \leq \mathrm{L} \leq \frac{\mathrm{T}_{\mathrm{F}}}{\mathrm{t}_{1}+\mathrm{t}_{2}}$ 
Poisson distribution [8] used for the generation of traffic is described in the traffic inter-arrival model in Figure 6. It states that nodes statistically generate traffic that is based on an exponentially distributed inter-arrival time. To test the algorithm's behavior for different arrival rates assume that the inter-arrival time between two successive packets be the random variable $\mathrm{T}$, the probability density function (PDF) for the inter-arrival time of Poisson traffic follows the exponential distribution that can be written as [11]:

$\mathrm{f}_{\mathrm{T}}(\mathrm{t})=\lambda \mathrm{e}^{-\lambda \mathrm{t}}$

Where, $\lambda$ is the average data rate, $\sigma$ is maximum burst rate and $\alpha$ is the average packet length in bits. The inter-arrival time distribution is modified to get the shifted exponential distribution can be described as:

$\lambda_{\mathrm{T}}(\mathrm{t})=\mathrm{b} \mathrm{e}-\mathrm{b}(\mathrm{t}-\mathrm{a}) \quad$ for $\mathrm{t} \geq \mathrm{a}$

Where, a: Position parameter which represents the minimum time between adjacent packets, $\mathrm{a}>0$ and $\mathrm{b}$ : The shape parameter that describe how fast the exponential function decays with time. The values of $a$ and $b$ for a source with parameters $\lambda, \sigma$ and $\alpha$ can be evaluated as:

$\mathrm{a}=\frac{\alpha}{\sigma}$

$\mathrm{b}=\frac{1}{\alpha} \times \frac{\sigma \lambda}{\sigma-\lambda}$

$\lambda=\frac{1}{\mathrm{~T}}$

$\sigma=\frac{1}{\mathrm{~T}-\Theta}$

$\Theta$ is a constant value between 1 and $\mathrm{T}-1$, but for simulation it has taken 1 . The average inter-arrival time $\mathrm{T}$ of the packets in this simulation was taken from $2-10 \mathrm{~s}$ and the average packet length $\alpha$ was assumed to be fixed with only 38 bytes as most of the wireless networks have a small packet size.

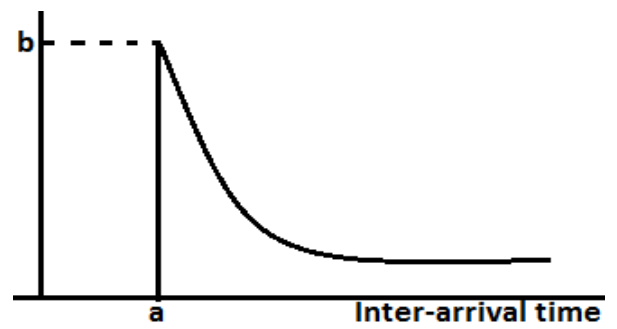

Figure 6: Biased exponential distributed with the two design parameters $a$ and $b$

\section{SIMULATION AND RESULTS}

The traffic is first generated for all the nodes in the networks for the entire simulation time i.e., 200 s. Each packets generated from any node is stored in the transmit buffer and is allocated at arrival time, destination node address and reservation slot address. These are required to calculate the time and the energy required to send that packet to its destination. The listen period is $300 \mathrm{~ms}$ for ML-MAC with L layers. The size of a data packet takes only $20 \mathrm{~ms}$ to send in a typical radio channel and the clock-drift delay is $0.5 \mathrm{~ms}$. The traffic is analyzed by the time index and checking for packets until the end of simulation. Here, the time index is set to be frame duration/1000, i.e., frames are split into 1000 slots. The total energy consumed by each node over the entire simulation time is determined by evaluating the time of each node spends in the three routines, i.e., listen, transmit, sleep.

$\mathrm{E}_{\text {total }}=\left(\mathrm{T}_{\text {listen }} \times \mathrm{P}_{\text {listen }}\right)+\left(\mathrm{T}_{\text {transmit }} \times \mathrm{P}_{\text {transmit }}\right)+\left(\mathrm{T}_{\text {sleep }} \times \mathrm{P}_{\text {sleep }}\right)$

Then the total energy consumed by the node is calculated by multiplying the total time nodes spend in each routine with the amount of power consumed in that routine. In PSO optimization, listen time, transmit time and sleep time are optimized at different layers. PSO optimized result the energy consumption for ML-MAC at average inter-arrival time $\mathrm{T}=$ 5 s, i.e., $\lambda=0.2$ packets $/ \mathrm{s}$ is shown in Figure 7 . Here the energy consumption decreases rapidly up to $\mathrm{L}=5$ and after five layers, energy consumption is not significant as most of the packets are destined to others layers and the nodes spend more time waking up at different schedules. Also, this increases the number of control packets that consumes more energy. It is the total energy consumed in a node for the whole simulation time, as the number of layers $\mathrm{L}$ is increased from 1 to 10 layers using non-coherent traffic.

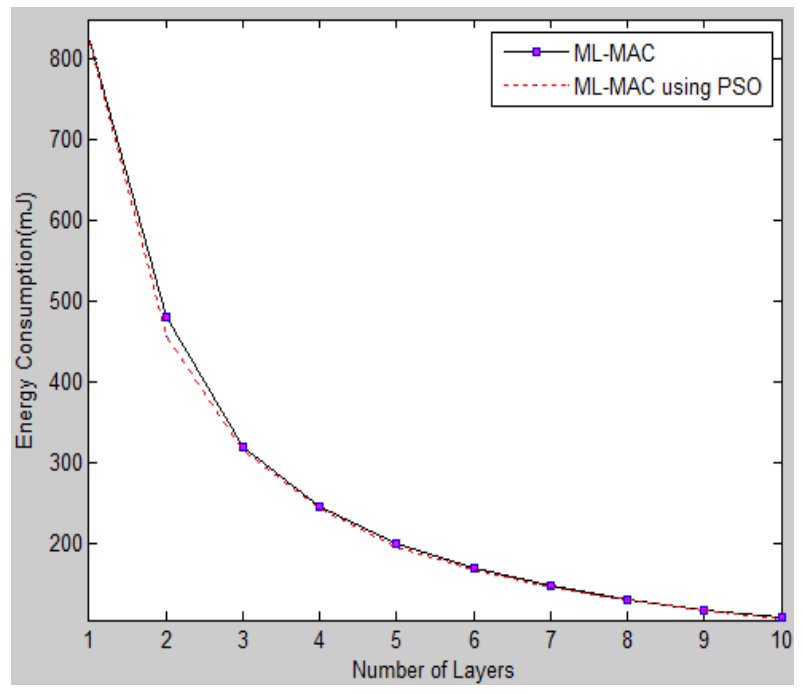

Figure 7: Energy consumption per node for ML-MAC in the non-coherent case

PSO optimized results for ML-MAC with delays is compared with ML-MAC without delays in Figure 8. If a node can transmit packets to any other nodes in a different access layer including the same access layer is called non-coherent traffic. In the simulation 4 types of delay are taken, i.e., transmission delay, maximum response time, queuing delay, clock drift delay. It results when the traffic is heavy, i.e., the message inter-arrival time is less than about 5s, ML-MAC without delay consumes $25 \%$ less delay than ML-MAC with delay and when the traffic is light, i.e., the message inter-arrival time is greater than about $5 \mathrm{~s}$, ML-MAC without delay consumes $7 \%$ less delay than ML-MAC with delay. 


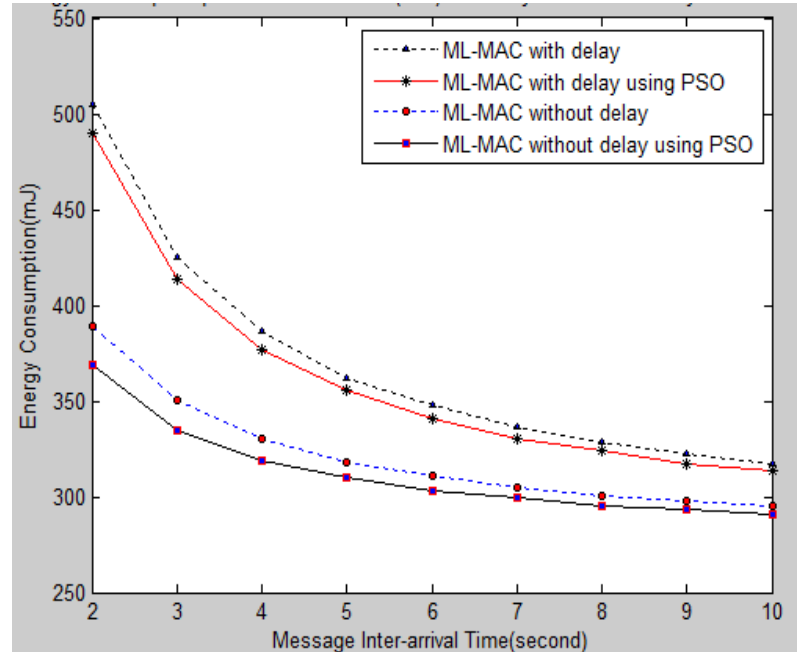

Figure 8: Total energy consumption per node for ML$\operatorname{MAC}(L=3)$ for the non-coherent case

In ML-MAC taking the number of layer constant at $\mathrm{L}=3$, maximum energy, average energy and minimum energy with delays is compared with maximum energy, average energy and minimum energy without delay in Figure 9. When a packet is sent, there should consider the clock-drift delay, transmission delay, maximum response delay and queuing delays. For 100 nodes the total energy is calculated for each. Among them the maximum, minimum and average energy is found for each inter-arrival time, 2-10 s.

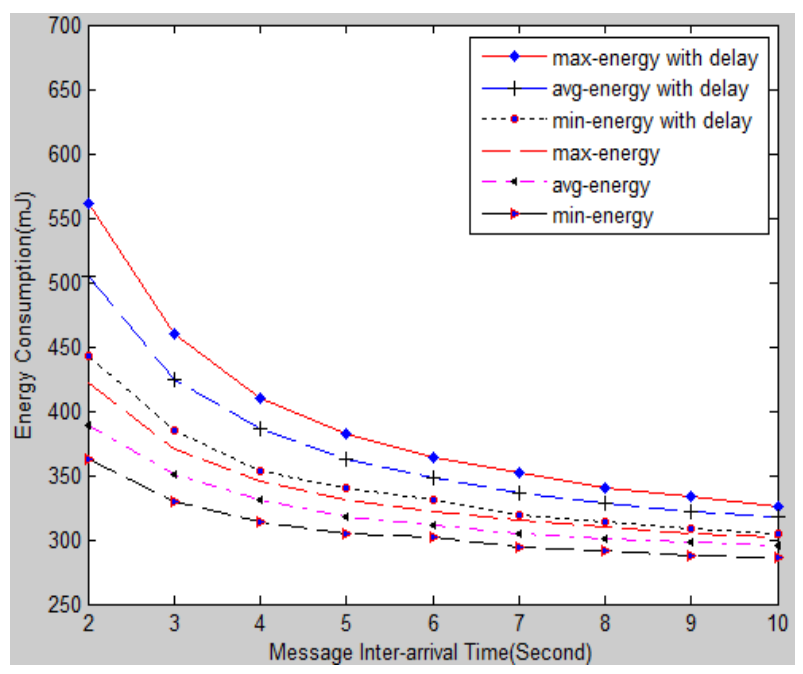

Figure 9: Maximum, Average \& Minimum energy consumption per node for ML-MAC algorithm $(\mathrm{L}=3)$ in the non-coherent case

Average delay for all packets in ML-MAC without PSO is compared with the PSO optimized value keeping the traffic constant at $\lambda=0.2$ packets/s in Figure 10. Delay is the sum of the time a packet may encounter in the transmit buffer and the time required to send that packet. So queuing delay is the dominant part that affects the delay. It is evaluated by subtracting the time a packet is received by the destination from the time it was generated. If the number of layers is less than three, the delay would increase rapidly. But, when more layers are added, then packets will not encounter more delay as they are usually buffered for the next frame cycle.

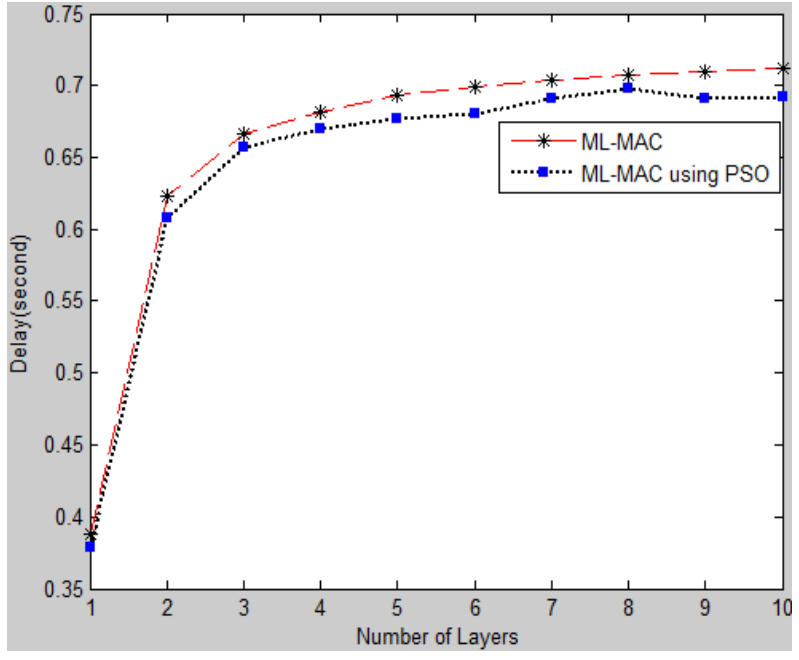

Figure 10: Average delay for all packets sent for ML$\mathrm{MAC}$ in the non-coherent case

In ML-MAC, latency means delay, the time of a packet is stored in node buffer until it is sent successfully without collision to its destination. Therefore, the delay $[13,14]$ here is composed of two components, i.e., Time waiting at output link for transmission is called queuing delay which depends on congestion level of router or traffic load and transmission delay is defined by channel bandwidth, packet length and caused by the data-rate of the link.

PSO optimized results the average delay of ML-MAC taking 4 types of delay is compared with the average delay of MLMAC taking 2 types of delay in Figure 11. ML-MAC of 4 delays in the non-coherent case has a longer delay than MLMAC of 2 delays. When the message inter-arrival time is less than 5s, ML-MAC of 2 delays, i.e., transmission delay \& queuing delay consume 19\% less delay than ML-MAC of 4 delays, i.e., queuing delay, transmission delay, clock-drift delay and maximum response delay. When the message interarrival time is greater than 5s, ML-MAC of 2 delays, i.e., transmission delay \& queuing delay consume $18 \%$ less delay than ML-MAC taking 4 delays, i.e., queuing, transmission, clock-drift and maximum response delay.

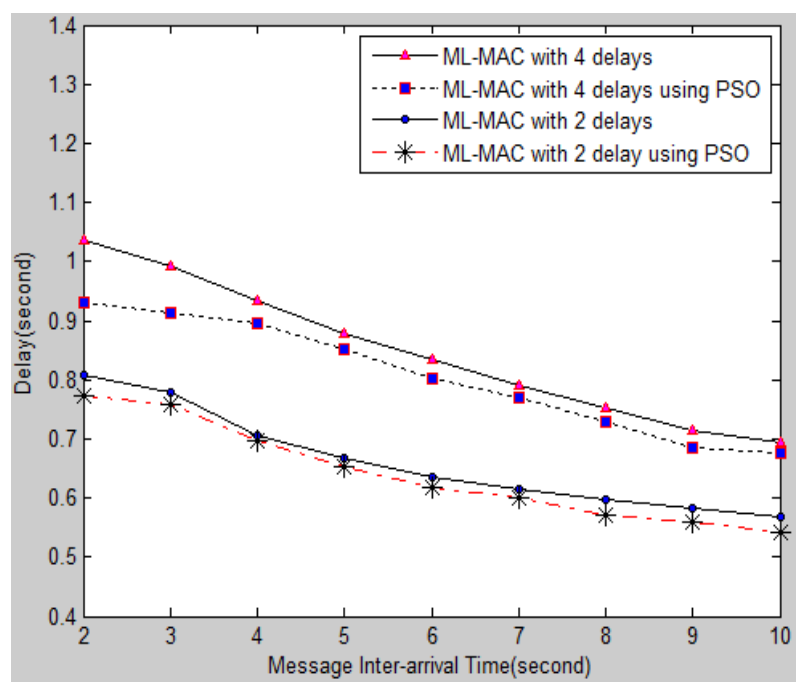

Figure 11: Average delay for all packets sent for ML$\operatorname{MAC}(L=3)$ in the non-coherent case 
PSO optimized results the probability of collision for MLMAC keeping the message inter-arrival time constant at $5 \mathrm{~s}$ in Figure 12. It results when traffic is heavy, more packets are generated and when the message inter-arrival time is above $5 \mathrm{~s}$, then traffic is light, less packets are generated. The probability of collision is defined as the ratio between number of collision and total number of packets. It can be defined by the below equation.

Probability of collision $=\frac{\text { Number of collision }}{\text { Total number of packets }}$

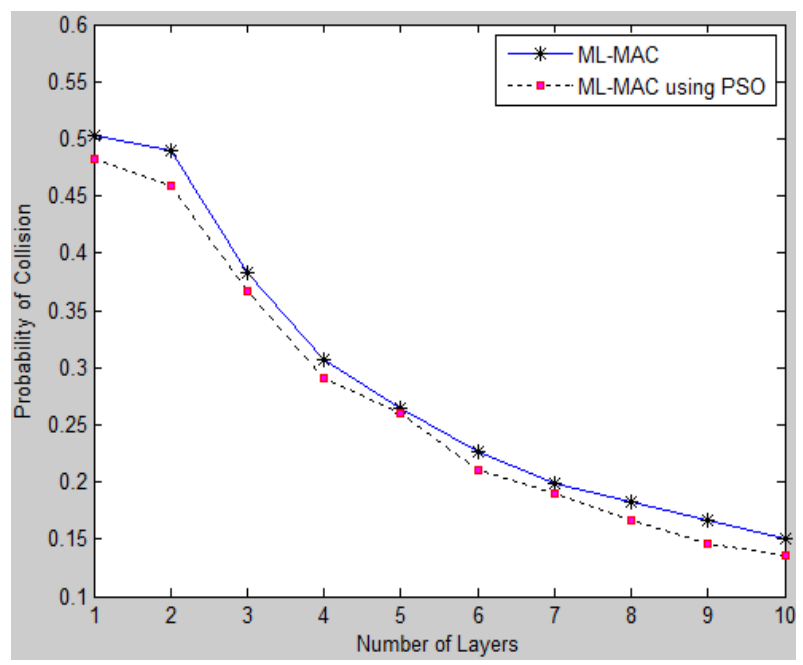

Figure 12: Probability of collisions for ML-MAC in the non-coherent case, traffic is fixed: $\lambda=0.2$ packets $/ \mathrm{s}$

\section{CONCLUSION}

PSO optimized results for energy consumption in ML-MAC based wireless sensor networks are presented here. In ML-MAC scheme, nodes are distributed in number of layers in order to minimize the idle listening time. PSO optimized simulation results for the energy consumption and average delay for ML-MAC with delay and without delay are compared. PSO optimized results show better performance in energy consumption. Comparison of energy consumption using present method with communication controlled MAC protocol for WSN is one of the future works. The idea of present optimization may be used for communication controlled slotted protocols for WSN. Further research work on this topic may be extension of this optimization method for energy consumption for varying traffic load.

\section{REFERENCES}

[1] Ye, W., Heidemann, J., Estrin, D. 2002. An energyefficient MAC protocol for wireless sensor networks. IEEE INFOCOM 2002, Vol.3, 1567 - 1576.Ye, W.,
Heidemann, J., Estrin, D. 2004. Medium access control with coordinated adaptive sleeping for wireless sensor networks. IEEE/ACM Transactions on Networking, Vol. 12, No.3, 493-506.

[2] Koutsakis, P. 2006. On increasing energy conservation for wireless sensor networks. Technical University of Crete. ICWMC'06 (July 2006), 4-14.

[3] Sohraby, K., Minoli, D., and Znati, T. Wireless sensor networks, Technology, protocols, and Applications, Second Edition, Wiley \& Sons., 4435-36/7.

[4] Yadav, R., Varma, S., and Malaviya, N. 2009. A survey of MAC protocols for wireless sensor networks. UbiCC Journal (August 2009) Volume 4, Number 3, 827-833.

[5] Singh, S., Raghavendra, C. S. 1998. PAMAS-power aware multi-access protocol with signalling for ad-hoc networks. ACM SIGCOMM Computer Communication Review (July 1998). 5-26.

[6] Jurdak, R., Lopes, C. V., Baldi, P. 2004. A survey, classification and comparative analysis of medium access control protocols for ad hoc networks. IEEE Communications Surveys \& Tutorials, 2-16.

[7] Wireless LAN medium access control (MAC) and physical layer (PHY) specification. IEEE std. 802.11; 1999.

[8] Kennedy, J., Eberhart, R., 1995 Particle swarm optimization, Proc. IEEE Int. Conf. Neural Networks, Piscataway, NJ, 1942-1948.

[9] Latiff, N. M. A., Tsimenidis, C. C., and Sharif, B. S. 2007. Energy-aware clustering for wireless sensor networks using particle swarm optimization. 18th Annual IEEE International Symposium on Personal, Indoor and Mobile Radio Communications, PIMRC'07, 1-5.

[10] Jha, M. K., Pandey, A. K., Pal, D., and Mohan, A. 2011. An energy efficient multi-layer MAC (ML-MAC) protocol for wireless sensor networks. International Journal of Electronics and Communications (AEÜ) 65, 209-216.

[11] ASH transceiver TR1000 data sheet, "RF Monolithic Inc. [Online]. http://www.rfm.com.

[12] Premkumar, M., Kathiravan, M., and Thirukkumaran, R. 2012. A novel MAC algorithm for energy aware wireless sensor networks. International Journal of Computer Applications, Vol.58, No.5, 1-4.

[13] Hameed, S. A., Shaaban, E. M., Faheem, H. M., and Ghoniemy, M. S. 2009. Mobility-aware MAC protocol for delay-sensitive wireless sensor networks. ICUMT '09, $1-8$. 